12. Hunter, C.A. 2005. New IL-12-family members: IL-23 and IL-27, cytokines with divergent functions. Nat. Rev. Immunol. 5:521-531.

13. McKenzie, B.S., Kastelein, R.A., and Cua, D.J. 2006. Understanding the IL-23-IL-17 immune pathway. Trends Immunol. 27:17-23.

14. Aggarwal, S., Ghilardi, N., Xie, M.H., de Sauvage, F.J., and Gurney, A.L. 2003. Interleukin-23 promotes a distinct CD4 $\mathrm{T}$ cell activation state characterized by the production of interleukin-17. J. Biol. Chem. 278:1910-1914.

15. Yen, D., et al. 2006. IL-23 is essential for T cellmediated colitis and promotes inflammation via IL-17 and IL-6. J. Clin. Invest. 116:1310-1316. doi:10.1172/JCI21404.

16. Strober, W., Fuss, I.J., and Blumberg, R.S. 2002. The immunology of mucosal models of inflammation. Annu. Rev. Immunol. 20:495-549.

17. Chen, Y., et al. 2006. Anti-IL-23 therapy inhibits multiple inflammatory pathways and ameliorates autoimmune encephalomyelitis. J. Clin. Invest. 116:1317-1326. doi:10.1172/JCI25308.

18. Cua, D.J., et al. 2003. Interleukin-23 rather than interleukin-12 is the critical cytokine for autoimmune inflammation of the brain. Nature 421:744-748.

19. Murphy, C.A., et al. 2003. Divergent pro- and antiinflammatory roles for IL-23 and IL-12 in joint autoimmune inflammation. J. Exp. Med. 198:1951-1957.

20. Afkarian, M., et al. 2002. T-bet is a STAT1-induced regulator of IL-12R expression in naive CD4+ T cells. Nat. Immunol. 3:549-557.

21. Wang, J., et al. 2006. Transcription factor T-bet regulates inflammatory arthritis through its function in dendritic cells. J. Clin. Invest. 116:414-421. doi:10.1172/JCI26631.

22. Parham, C., et al. 2002. A receptor for the heterodimeric cytokine IL-23 is composed of IL-12Rbeta 1 and a novel cytokine receptor subunit, IL-23R. J. Immunol. 168:5699-5708.

23. Kollias, G., and Kontoyiannis, D. 2002. Role of TNF/TNFR in autoimmunity: specific TNF receptor blockade may be advantageous to anti-TNF treatments. Cytokine Growth Factor Rev. 13:315-321.

24. Matsuki, T., Nakae, S., Sudo, K., Horai, R., and Iwakura, Y. 2006. Abnormal T cell activation caused by the imbalance of the IL-1/IL-1R antagonist system is responsible for the development of experimental autoimmune encephalomyelitis. Int. Immunol. 18:399-407.

25. Samoilova, E.B., Horton, J.L., Hilliard, B., Liu, T.S., and Chen, Y. 1998. IL-6-deficient mice are resistant to experimental autoimmune encephalomyelitis: roles of IL- 6 in the activation and differentiation of autoreactive T cells. J. Immunol. 161:6480-6486.

26. Veldhoen, M., Hocking, R.J., Atkins, C.J., Locksley, R.M., and Stockinger, B. 2006. TGFbeta in the context of an inflammatory cytokine milieu supports de novo differentiation of IL-17-producing T cells. Immunity. 24:179-189.

27. Shtrichman, R., and Samuel, C.E. 2001. The role of gamma interferon in antimicrobial immunity. Curr. Opin. Microbiol. 4:251-259.

28. Happel, K.I., et al. 2005. Divergent roles of IL-23 and IL-12 in host defense against Klebsiella pneumoniae. J. Exp. Med. 202:761-769.

29. Stark, M.A., et al. 2005. Phagocytosis of apoptotic neutrophils regulates granulopoiesis via IL-23 and IL-17. Immunity. 22:285-294.

\title{
Role of caveolin-1 in the regulation of the vascular shear stress response
}

\author{
Philippe G. Frank ${ }^{1,2,3}$ and Michael P. Lisanti²,3 \\ ${ }^{1}$ Department of Urology and ${ }^{2}$ Departments of Molecular Pharmacology and Medicine and the Albert Einstein \\ Diabetes Research and Training Center (DRTC), Albert Einstein College of Medicine, New York, New York, USA. \\ ${ }^{3}$ Department of Cancer Biology, Kimmel Cancer Center, Thomas Jefferson University, Philadelphia, Pennsylvania, USA.
}

\begin{abstract}
In blood vessels, endothelia are submitted to constant shear effects and are, under normal conditions, capable of responding to any variation in hemodynamic forces. Caveolae -50 - to 100 -nm plasma membrane invaginations present at the surface of terminally differentiated cells and particularly enriched in ECs - are composed of a high sphingolipid and cholesterol content and the protein caveolin-1 (Cav-1). Previous studies have suggested that caveolae and endothelial Cav-1 may regulate the vascular response to altered shear stress. In this issue of the JCI, Yu et al. have examined the role of Cav-1/caveolae in the regulation of flow-induced alterations (i.e., mechanotransduction) in vessels from wild-type mice, Cav-1-deficient mice, and Cav-1-deficient mice re-expressing Cav-1 only in ECs. Their data suggest that caveolae/Cav-1 may act as sensors of altered shear stress and that they also organize the signaling response in stimulated ECs (see the related article beginning on page 1284).
\end{abstract}

\section{Shear stress: an important regulator of endothelial cell function}

In the vasculature, blood vessels must respond rapidly to any external stimuli and especially to any physical change related to modifications in shear stress, which is a function of the blood viscosity and the velocity gradient at the arterial

Nonstandard abbreviations used: Cav-1, caveolin-1.

Conflict of interest: The authors have declared that no conflict of interest exists.

Citation for this article: J. Clin. Invest. 116:1222-1225 (2006). doi:10.1172/JCI28509. wall. In this context, blood vessels need to adapt and adjust their luminal diameters and their physical properties. ECs are the primary targets of these changes, as they are the first cell type exposed to these forces. One of the earliest findings that suggested an important role for ECs in this process was the observation of the ability of ECs to reorient and change shape during exposure to shear stress conditions. When submitted to steady laminar shear stress, ECs reorient in the direction of the flow and become remarkably elongated. These observations were made both in vitro (1) and in vivo $(2,3)$. These findings have suggested that ECs can respond and adapt to changes in blood flow. In fact, ECs act as sensors to transduce hydrodymanic forces. Not only does the morphology of ECs change, but other important signaling pathways have been shown to be regulated in response to altered shear stress. Several studies have shown that under laminar shear stress, the rate of EC proliferation is reduced compared with static conditions $(4,5)$. Oscillating and/or disturbed conditions have major effects on the pathology of the vasculature. In this regard, atherosclerotic lesions have been shown to develop primarily at sites of disturbed or altered blood flow, i.e., at bifurcations, branch ostia, and curved regions (6). Under oscillating and/or disturbed conditions, EC proliferation is increased compared with cells submitted to laminar shear stress and may allow ECs to repair injuries $(7,8)$. Other important regulatory pathways have now been shown to be activated under shear stress conditions (9-11). These pathways include those involved in changes in endothelial apoptotic, migratory, and permeability prop- 


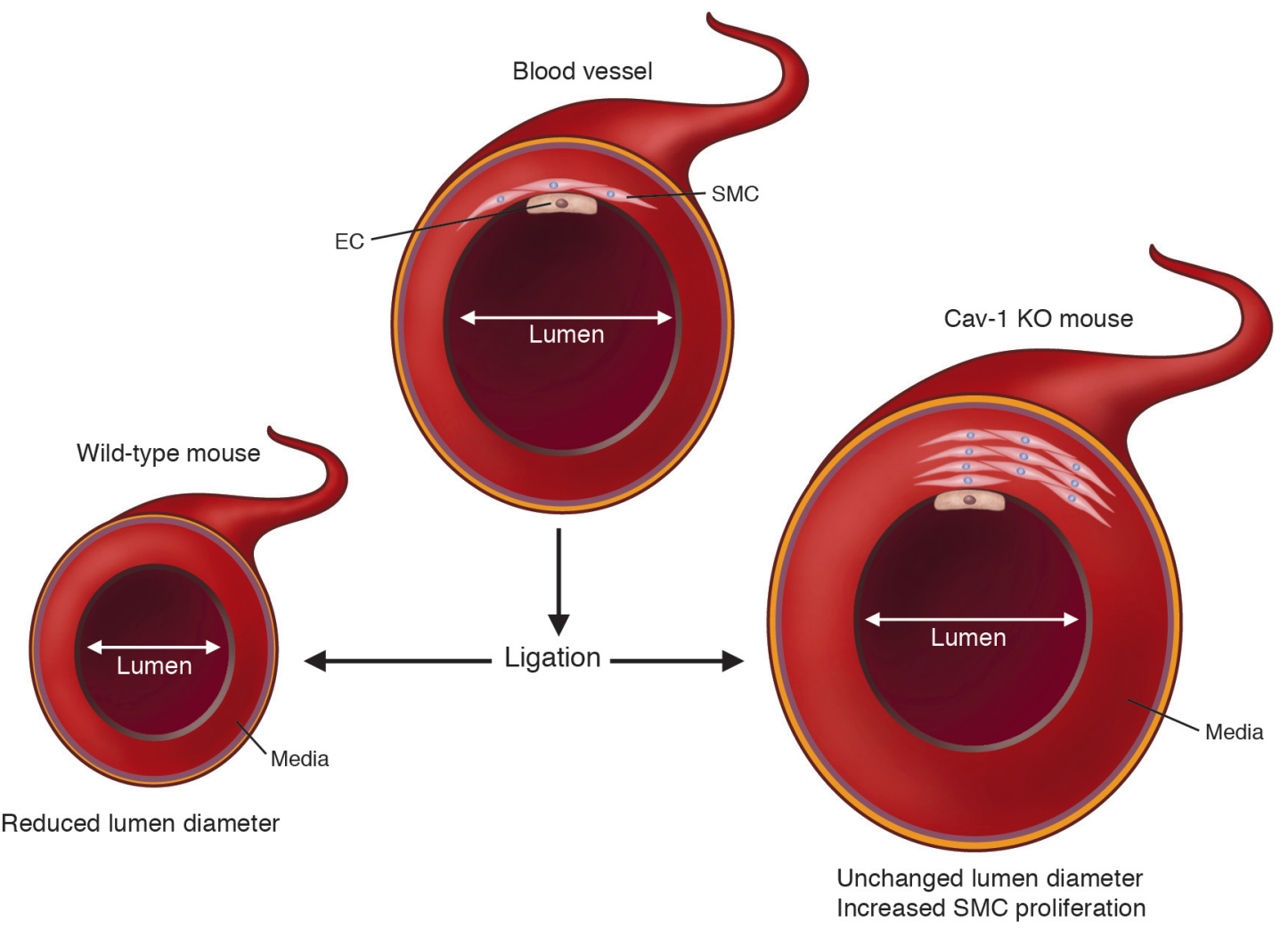

Figure 1

Cav-1 deficiency is associated with an altered response to modified shear stress conditions. In this issue of the JCl, Yu et al. (20) have demonstrated that Cav-1 deficiency is associated with a reduced vascular response upon the alteration of shear stress conditions. Accordingly, reduced blood flow in the common carotid artery (due to ligation) was associated with reduced vessel lumen diameter in the case of wild-type animals. However, in Cav-1 KO mice, increased SMC proliferation was detected in the media but no change in the vessel lumen diameter was observed. Re-expression of Cav-1 in ECs alone was sufficient to eliminate this phenotype in Cav-1 KO mice.

erties, among others. Many signaling molecules have also been shown to be involved, including $\mathrm{PKC}$, focal adhesion kinase (FAK), s-Src, Rho family GTPases, PI3K, MAPKs, and the production of $\mathrm{NO}$ via eNOS (11).

\section{Caveolae and caveolin-1: roles in vascular regulation of the shear stress-induced response}

In order to properly respond to changes during conditions of shear stress, ECs rely on specific sensors such as integrins, ion channels, receptor tyrosine kinases, and caveolae. Caveolae are 50- to $100-n m$ plasma membrane invaginations that are characterized by the concentration of specific lipid components (sphingolipids/cholesterol) and the presence of the protein marker caveolin-1 (Cav-1) (12). In vivo, the EC cell type is highly enriched in caveolae and Cav-1. Caveolae have been shown to play an important role in the regulation of various signaling cascades as well as endothelial microvascular permeability (13). Consistent with these observations, important vascular abnormalities have been observed in Cav-1-deficient (Cav-1 $\mathrm{KO})$ mice. Interestingly, caveolae numbers are remarkably increased in ECs that are cultured under laminar shear stress conditions compared with static conditions (14-17). In addition, it has been reported that caveolae and Cav-1 move toward the upstream edge of ECs and are associated with sites of $\mathrm{Ca}^{2+}$ wave initiation (14). It is important to note that Cav-1 has been shown to regulate several signaling pathways that are activated upon exposure of ECs to altered shear stress $(11,18,19)$. Taken together, these observations are consistent with a role for Cav-1 in the regulation of the EC response to altered shear stress.

In a study in this issue of the JCI, Yu et al. (20) have examined the role of caveolae and Cav-1 in the regulation of flowinduced alterations using Cav-1 KO mice and Cav-1 KO mice overexpressing Cav-1 specifically in ECs. Overexpression of Cav-1 in ECs in Cav-1 KO mice offers an elegant approach for examining the specific role of endothelial Cav-1 in this process. To alter shear stress conditions in vivo, they ligated the left carotid artery for 14 days, thereby resulting in modified blood flow in the common carotid artery. This led to reduced lumen diameter in control animals but not in Cav-1 KO mice. In contrast, increased wall thickness, associated with enhanced cellular proliferation, was observed in Cav-1 KO mice (Figure 1). But the most important finding was that re-expression of Cav-1 in ECs was sufficient to prevent these changes in Cav-1 KO mice. As previously described $(21,22)$, acetylcholine-mediated arterial relaxation was enhanced and tended to increase vasodilation in Cav-1 KO arteries. Endotheliumderived NO has a profound effect on vessel tonicity and permeability. Within the 


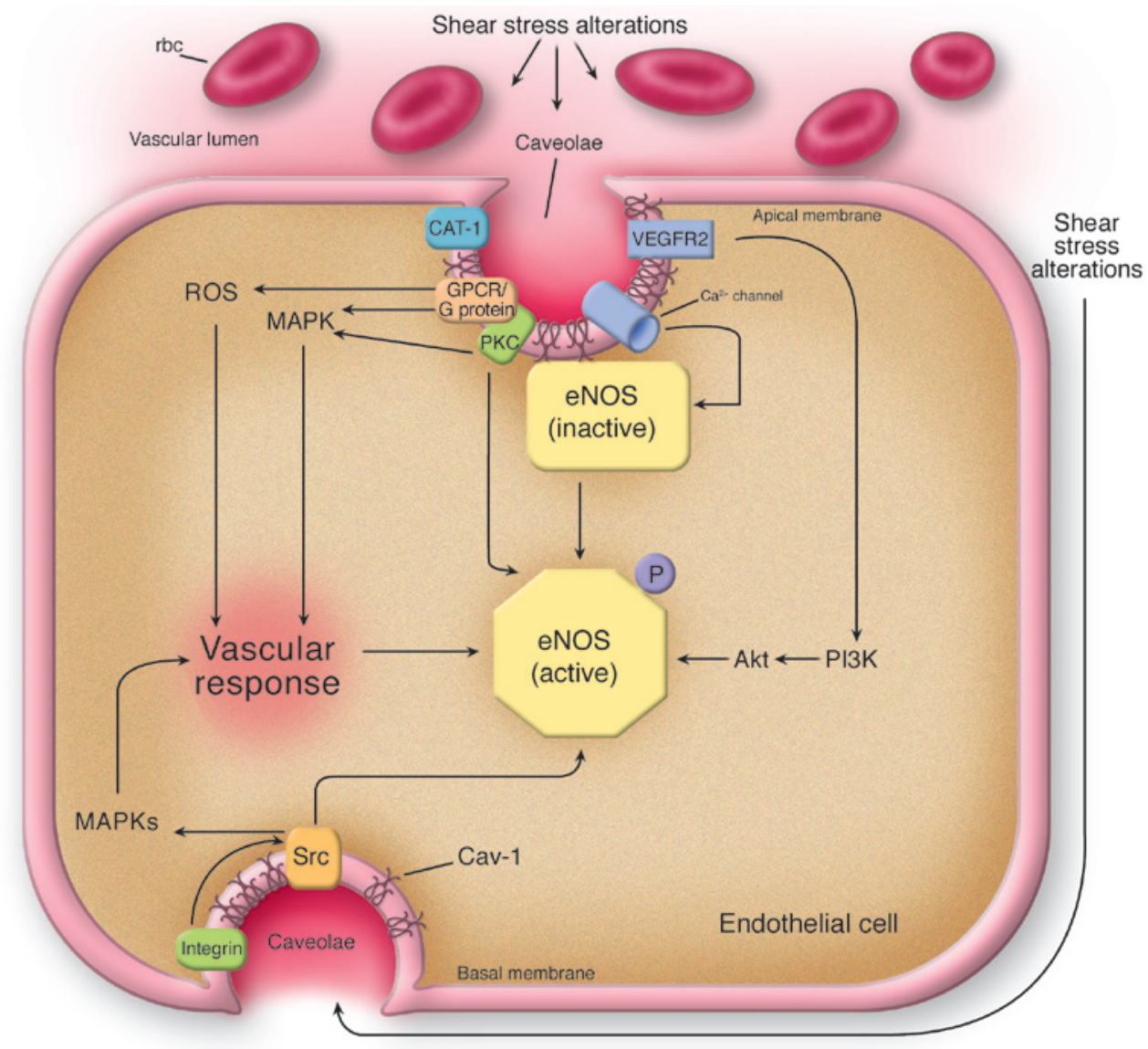

Figure 2

Cav-1-regulated signaling pathways in endothelial cells. According to Yu et al. (20), caveolae and Cav-1 appear to be involved in some of the earliest steps associated with the detection of altered shear stress conditions in blood vessels. In addition, the vascular response (see Figure 1) is induced via caveolae and Cav-1, as demonstrated for several signaling pathways. Cav-1 plays important roles in regulating eNOS function. In unstimulated cells, eNOS is maintained in an inactive state through its association with Cav-1. Upon stimulation (e.g., shear stress), Cav-1 and caveolae may allow for the proper organization of various signal transduction pathways or organize the different regulatory proteins necessary for rapid and efficient eNOS activation. Calcium channels and the arginine transporter CAT-1 are localized to caveolae and may therefore allow for efficient eNOS activation ( $\mathrm{Ca}^{2+}$ entry and dissociation from Cav-1 in the presence of calmodulin) and availability of substrate. Further activation is also possible via the PI3K and Akt signaling pathways, which enhance NO production following eNOS phosphorylation. Activation of the VEGFR2 signaling pathway is critical for PI3K/Akt activation, but other pathways appear to also mediate MAPK activation (ERK1/2 in particular). These pathways also involve G protein-coupled receptors (GPCRs) and G proteins. Shear stress is associated with increased oxidative stress conditions that lead to the production of ROS. Additionally, shear stress may affect EC interactions with extracellular matrix proteins. This may in turn activate integrin-mediated signaling pathways via caveolae. Src may also directly alter eNOS activity by inducing tyrosine phosphorylation (33). Note that only the major affected signal transduction pathways are shown.

endothelial plasma membrane, eNOS is highly enriched in caveolae (23). Optimal eNOS activity occurs when the interaction between eNOS and Cav-1 is competitively disrupted by calcium-calmodulin binding to eNOS $(24,25)$. In addition, eNOS may be activated by phosphorylation of serine 1176 (mouse eNOS) via activation of the Akt pathway $(26,27)$ during vascular remodeling using the same model (28). In the current study, Yu et al. (20) provide compelling evidence that Cav-1 KO carotids show reduced flow-dependant signaling and coupling of eNOS activity. In agreement with this hypothesis, acti- vation of eNOS via phosphorylation of Ser1176 was reduced in Cav-1 KO arteries. This deficient activation appeared to be due, at least in part, to the mislocalization of eNOS in a perinuclear compartment. Importantly, re-expression of endothelial Cav-1 in Cav-1 KO ECs was sufficient to reverse these effects.

This paper highlights the importance of the proper integration and coordination of the different signal transduction pathways within caveolae (Figure 2). While under basal conditions, Cav-1 can repress eNOS activity (Figure 2), it is now becoming clear that Cav-1 allows for the proper activation of eNOS activity upon stimulation of ECs (29). As previously suggested $(19,30)$, caveolae may act as platforms that hold signaling molecules associated with various signaling pathways in an inactive state. Upon stimulation (e.g., altered shear stress), signals may be rapidly transferred to downstream effectors to allow cells to respond quickly and efficiently. However, eNOS may not be the only protein affected in Cav-1 KO mice. Other important regulators of EC function, including VEGFR2, FAK, and integrins, may also play important roles in the phenotypes observed in Cav-1 KO mice. Additionally, several mol- 
ecules involved in calcium translocation have been localized to caveolae where they are active (31). Given the rapid changes in intracellular calcium concentrations in ECs under altered shear stress (32), caveolae and Cav-1 may be involved in the very early sensing events leading to the endothelial response.

These data suggest that endothelial caveolae and Cav-1 allow arteries to sense, organize, and mediate signal transduction, thereby giving arteries the ability to change their physical properties and to maintain/ regulate normal blood flow in the face of altered shear stress conditions.

Address correspondence to: Michael P. Lisanti or Philippe G. Frank, Kimmel Cancer Center, Departments of Cancer Biology and Biochemistry \& Molecular Biology, Thomas Jefferson University, 233 South 10th Street, Bluemle Life Sciences Building, Room 933, Philadelphia, Pennsylvania 19107, USA. Phone: (215) 503-9295; Fax: (215) 923-1098; E-mail: michael.lisanti@ jefferson.edu (M.P. Lisanti). Phone: (215) 503-9292; Fax: (215) 923-1098; E-mail: philippe.frank@jefferson.edu (P.G. Frank).

1. Dewey, C.F., Jr., Bussolari, S.R., Gimbrone, M.A., Jr., and Davies, P.F. 1981. The dynamic response of vascular endothelial cells to fluid shear stress. J. Biomech. Eng. 103:177-185.

2. Flaherty, J.T., et al. 1972. Endothelial nuclear patterns in the canine arterial tree with particular reference to hemodynamic events. Circ. Res. 30:23-33.

3. Langille, B.L., and Adamson, S.L. 1981. Relationship between blood flow direction and endothelial cell orientation at arterial branch sites in rabbits and mice. Circ. Res. 48:481-488.

4. Levesque, M.J., Nerem, R.M., and Sprague, E.A. 1990. Vascular endothelial cell proliferation in culture and the influence of flow. Biomaterials. 11:702-707.

5. Akimoto, S., Mitsumata, M., Sasaguri, T., and Yoshi- da, Y. 2000. Laminar shear stress inhibits vascular endothelial cell proliferation by inducing cyclindependent kinase inhibitor p21(Sdi1/Cip1/Waf1). Circ. Res. 86:185-190.

6. Cunningham, K.S., and Gotlieb, A.I. 2005. The role of shear stress in the pathogenesis of atherosclerosis. Lab. Invest. 85:9-23.

7. Davies, P.F., Remuzzi, A., Gordon, E.J., Dewey, C.F., Jr., and Gimbrone, M.A., Jr. 1986. Turbulent fluid shear stress induces vascular endothelial cell turnover in vitro. Proc. Natl. Acad. Sci. U. S. A. 83:2114-2117.

8. Langille, B.L., Reidy, M.A., and Kline, R.L. 1986. Injury and repair of endothelium at sites of flow disturbances near abdominal aortic coarctations in rabbits. Arteriosclerosis. 6:146-154.

9. Fisher, A.B., Chien, S., Barakat, A.I., and Nerem, R.M. 2001. Endothelial cellular response to altered shear stress. Am. J. Physiol. Lung Cell. Mol. Physiol. 281:L529-L533.

10. Langille, B.L. 2001. Morphologic responses of endothelium to shear stress: reorganization of the adherens junction. Microcirculation. 8:195-206.

11. Li, Y.S., Haga, J.H., and Chien, S. 2005. Molecular basis of the effects of shear stress on vascular endothelial cells. J. Biomech. 38:1949-1971.

12. Razani, B., and Lisanti, M.P. 2001. Caveolin-deficient mice: insights into caveolar function and human disease. J. Clin. Invest. 108:1553-1561. doi:10.1172/JCI200114611.

13. Hassan, G., Lisanti, M., and Frank, P. 2005. Caveolae and caveolins in the vascular system: functional roles in endothelia, macrophages, and smooth muscle cells. In Caveolae and lipid rafts: roles in signal transduction and the pathogenesis of human diseases. M.P. Lisanti and P.G. Frank, editors. Elsevier. 187-209.

14. Isshiki, M., et al. 2002. Sites of $\mathrm{Ca}(2+)$ wave initiation move with caveolae to the trailing edge of migrating cells. J. Cell Sci. 115:475-484.

15. Sun, R.J., Muller, S., Stoltz, J.F., and Wang, X. 2002. Shear stress induces caveolin-1 translocation in cultured endothelial cells. Eur. Biophys. J. 30:605-611.

16. Boyd, N.L., et al. 2003. Chronic shear induces caveolae formation and alters ERK and Akt responses in endothelial cells. Am. J. Physiol. Heart Circ. Physiol. 285:H1113-H1122.

17. Rizzo, V., Morton, C., DePaola, N., Schnitzer, J.E., and Davies, P.F. 2003. Recruitment of endothelial caveolae into mechanotransduction pathways by flow conditioning in vitro. Am. J. Physiol. Heart Circ. Physiol. 285:H1720-H1729.

18. Rizzo, V., Sung, A., Oh, P., and Schnitzer, J.E. 1998. Rapid mechanotransduction in situ at the luminal cell surface of vascular endothelium and its caveolae. J. Biol. Chem. 273:26323-26329.
19. Frank, P.G., Woodman, S.E., Park, D.S., and Lisanti, M.P. 2003. Caveolin, caveolae, and endothelial cell function. Arterioscler. Thromb. Vasc. Biol. 23:1161-1168.

20. Yu, J., et al. 2006. Direct evidence for the role of caveolin-1 and caveolae in mechanotransduction and remodeling of blood vessels. J. Clin. Invest. 116:1284-1291. doi:10.1172/JCI27100.

21. Drab, M., et al. 2001. Loss of caveolae, vascular dysfunction, and pulmonary defects in caveolin-1 gene-disrupted mice. Science. 293:2449-2452.

22. Razani, B., et al. 2001. Caveolin-1 null mice are viable but show evidence of hyperproliferative and vascular abnormalities. J. Biol. Chem. 276:38121-38138.

23. Shaul, P.W., et al. 1996. Acylation targets endothelial nitric-oxide synthase to plasmalemmal caveolae. J. Biol. Chem. 271:6518-6522.

24. Ju, H., Zou, R., Venema, V.J., and Venema, R.C. 1997. Direct interaction of endothelial nitric-oxide synthase and caveolin-1 inhibits synthase activity. J. Biol. Chem. 272:18522-18525.

25. Michel, J.B., Feron, O., Sacks, D., and Michel, T. 1997. Reciprocal regulation of endothelial nitricoxide synthase by $\mathrm{Ca}^{2+}$-calmodulin and caveolin. J. Biol. Chem. 272:15583-15586.

26. Dimmeler, S., et al. 1999. Activation of nitric oxide synthase in endothelial cells by Akt-dependent phosphorylation. Nature. 399:601-605.

27. Fulton, D., et al. 1999. Regulation of endotheliumderived nitric oxide production by the protein kinase Akt. Nature. 399:597-601.

28. Rudic, R.D., et al. 1998. Direct evidence for the importance of endothelium-derived nitric oxide in vascular remodeling. J. Clin. Invest. 101:731-736.

29. Sbaa, E., Frerart, F., and Feron, O. 2005. The double regulation of endothelial nitric oxide synthase by caveolae and caveolin: a paradox solved through the study of angiogenesis. Trends Cardiovasc. Med. 15:157-162.

30. Lisanti, M.P., Scherer, P., Tang, Z.-L., and Sargiacomo, M. 1994. Caveolae, caveolin and caveolin-rich membrane domains: a signalling hypothesis. Trends Cell Biol. 4:231-235.

31. Isshiki, M., Ying, Y.S., Fujita, T., and Anderson, R.G. 2002. A molecular sensor detects signal transduction from caveolae in living cells. J. Biol. Chem. 277:43389-43398.

32. Ando, J., Komatsuda, T., and Kamiya, A. 1988. Cytoplasmic calcium response to fluid shear stress in cultured vascular endothelial cells. In Vitro Cell. Dev. Biol. 24:871-877.

33. Fulton, D., et al. 2005. Src kinase activates endothelial nitric-oxide synthase by phosphorylating Tyr-83. J. Biol. Chem. 280:35943-35952. 